\title{
Artificial Material Integrated Ultra-wideband Tapered Slot Antenna for Gain Enhancement with Band Notch Characteristics
}

\author{
Rahul SINGHA, Vakula DAMERA \\ Dept. of Electronics and Communication Engineering, National Institute of Technology Warangal, 506004, India \\ naoremrahul5488@hotmail.com,vakula@nitw.ac.in \\ Submitted March 20, 2017 / Accepted October 26, 2017
}

\begin{abstract}
The gain of the ultra-wideband tapered slot antenna (TSA) is enhanced by using broadband artificial material with band notch characteristics. The proposed artificial material unit cell is designed by fabricating nonresonant three $S$-shaped parallel metallic line on single side of the dielectric substrate which provides a longer current path compared to the parallel-line structure. The proposed S-shaped structure is printed on the top side of the tapered slot antenna in the extended substrate periodically. The effective refractive index of the artificial material is lower than antenna substrate and phase velocity in the region of artificial material is much higher than the other region. Therefore, the proposed artificial material acts like a beam focusing lens. The band notch at $5.5 \mathrm{GHz}$ is achieved by creating a split ring resonator (SRR) slot near the balun. The basic and artificial material loaded TSAs are fabricated and the measurement results show that the gain of the basic antenna has been increased by 1.6 dBi. At the same time, the proposed antenna achieves a VSWR below 2 from 3 to $11 \mathrm{GHz}$ except at $5.5 \mathrm{GHz}$ with a notch band from 5.1 to $5.8 \mathrm{GHz}$ for band rejection of wireless local area network (WLAN) application.
\end{abstract}

\section{Keywords}

Tapered slot antenna, artificial material, split ring resonator, wireless local area network (WLAN)

\section{Introduction}

The traditional Vivaldi antenna is well known for its broadband characteristics with light weight, low cost, ease of fabrication and capable of integration with other electronics systems. This antenna has another name called tapered slot antenna (TSA) [1]. Due to the end fire radiation, TSAs are used to provide a directional radiation pattern with simple structures. These types of antennas are widely used in both military and medical applications, e.g. microwave imaging, see through-wall (STW), ground penetrating radar, tumor detection, cancer detection etc.
According to the antenna theory, tapered slot antenna has infinite bandwidth [1]. Bandwidth is proportional to the length and aperture of antenna. Hence, TSA is a good candidate for ultra-wideband (UWB) application. In case of printed antennas, it is difficult to provide high gain as well as stable radiation pattern. In order to overcome these problems, different techniques exist such as antenna array [2], electromagnetic band gap (EBG) material [3], genetic algorithm optimization techniques [4] etc. But the size and circuit complexity of the antenna will increase.

In the past several years, artificial materials are studied by scientists and engineers because of some special electromagnetic (EM) properties which are very difficult to find in nature. According to the material properties and arrangements of meta-atoms (unit cells), an EM wave is able to have enhanced emissions [5], clear imaging [6], invisible cloaking [7], EM tunneling [8] and absorbers [9]. Among all artificial materials, the low or zero index metamaterial (LIM or ZIM) is widely applicable for its beam focusing properties. The zero-index metamaterial (ZIM) is a good option for all broadband antennas. The lens can help to improve the radiation pattern and gain of any antenna over broader frequency range.

Under UWB frequency band, WIMAX and WLAN bands are also allotted which will be the cause of serious electromagnetic interference (EMI). To remove these interferences, a complementary split ring resonator (CSRR) slot is applied in the Vivaldi antenna [10]. But the overall gain of the antenna is reduced towards the high frequencies. Moreover, the band notch is shown from $4.8 \mathrm{GHz}$ to $5.4 \mathrm{GHz}$, which has no practical importance. Split ring resonator (SRR) loaded coplanar waveguide (CPW) fed ultrawideband circular monopole antenna is designed with frequency notch characteristics in [11]. Unfortunately, the gain of the antenna is reduced towards the high frequencies. The gain is $-7 \mathrm{dBi}$ at notch frequency of $6.39 \mathrm{GHz}$, which has no practical importance. To enhance the gain of an ultrawideband (UWB) antenna, multioctave dual-layer frequency selective surface (FSS) reflector is designed in [12]. The antenna is more directive in range $7-14 \mathrm{GHz}$, with a gain of about $9 \mathrm{dBi}$ with $\pm 0.5 \mathrm{~dB}$ variation. But the 
circuit complexity enhances with large size of dual layer FSS. So, the overall size of the antenna is increased with high side lobe in radiation pattern.

A novel band notch technique is introduced in the elliptically tapered slot antenna with arc-shaped parasitic strip printed inside the circular slot (balun) [15]. This design achieves minimization of physical size and the signal is rejected at 5-6 GHz. But the measured gain is varied at $4 \mathrm{~dB}$ over the operating frequency band and suppressed up to $-13 \mathrm{dBi}$ at $5.5 \mathrm{GHz}$. To overcome the low gain problem, zero-index metamaterial is designed to enhance the gain of the Vivaldi antenna and CSRR slot is used to suppress WLAN/WiMAX band [16]. The notch is created at $4.8 \mathrm{GHz}$ by using zero-index metamaterial (ZIM). The gain of the antenna is $3 \mathrm{dBi}$ at $4.8 \mathrm{GHz}$ by using ZIM in Vivaldi antenna. In conclusion, the CSRR effect is not sufficient to create a negative gain over a notch frequency. Recently, two different SRR geometries, square SRR (S-SRR) and hexagonal SRR (H-SRR), are used to obtain the frequency notches [17]. The measured gain is drastically reduced of $4.46 \mathrm{dBi}$ at $6.3 \mathrm{GHz}$ for the S-SRR and $-3.9 \mathrm{dBi}$ at $7.1 \mathrm{GHz}$ for the H-SRR resonant structures. But the gain is reduced at high frequency by using both resonators. In short, the gain at notch frequency and other operating frequency is not improved at the same time.

In this paper, a broadband artificial material integrated TSA with SRR slot is presented to enhance gain with band notch characteristics. The artificial material is integrated in tapered slot area of the basic TSA and the peak gain of antenna is increased from 9.4 to $10.9 \mathrm{dBi}$ at $10 \mathrm{GHz}$. The SRR is etched near the excitation area or input aperture of the antenna which is responsible for notch frequency at $5.5 \mathrm{GHz}$ and WLAN band is suppressed.

\section{Design and Characteristics of Broadband Artificial Material}

The unit cell artificial material is designed with nonresonant parallel S-shaped metallic structures i.e. placed side by side as shown in Fig. 1(a). Resonant metamaterials have dispersion properties over a narrow band. So, resonant metamaterials are not suitable for UWB application. Hence, a non-resonant metamaterial is used in this paper. The proposed material is designed on the Rogers RO4003 (dielectric constant, $\varepsilon_{\mathrm{r}}=3.55$ ) with substrate thickness, $t=0.8 \mathrm{~mm}$ and loss tangent of 0.0027 . The metamaterial unit cell dimension has been taken as $l_{\mathrm{x}}=l_{\mathrm{y}}=\lambda / 5.3$ at $6 \mathrm{GHz}$ where $\lambda=c / f \cdot \sqrt{\varepsilon_{\mathrm{r}}}$.

To extract the values of effective parameter, the scattering object is replaced by a homogeneous medium i.e. the wavelength within the medium becomes large in terms of the unit cell size [14]. To evaluate the scattering parameters, it is necessary to define periodic boundary conditions of the unit cell. The perfect electric conductor (PEC) and perfect magnetic conductor (PMC) boundary conditions

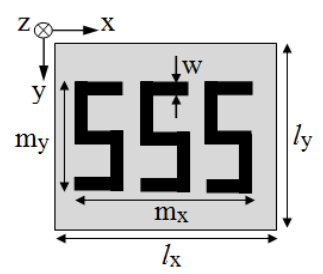

(a)

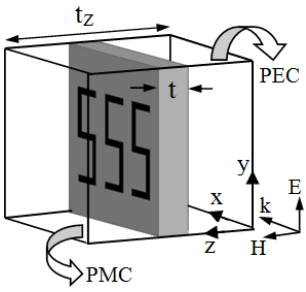

(b)
Fig. 1. The geometry of unit cell artificial material: (a) Unit cell artificial material. (b) The simulation model. The dimensions of parameters are $l_{\mathrm{x}}=l_{\mathrm{y}}=5 \mathrm{~mm}, m_{\mathrm{x}}=$ $2.8 \mathrm{~mm}, m_{\mathrm{y}}=4 \mathrm{~mm}$ and $w=0.2 \mathrm{~mm}, t=0.8 \mathrm{~mm}$ and $t_{\mathrm{z}}=5 \mathrm{~mm}$.

are indicated in Fig. 1(b). The electric and magnetic polarizations are along the $\mathrm{y}$ and $\mathrm{z}$-axes, respectively. The EM wave will propagate along $\mathrm{X}$-axis.

From the effects of electromagnetic properties in the medium, the magnitude and phase of S-parameters are calculated. The effective parameters of the proposed material are extracted from the S-parameters. The proposed artificial material parameters such as effective permittivity $\left(\varepsilon_{\mathrm{y}}\right)$ and permeability $\left(\mu_{\mathrm{z}}\right)$ are obtained via retrieval formula as described in [18]. The effective wave impedance $(z)$ and the effective refractive index $(n)$ for the given metamaterial unit cell are obtained from the following equations [18]:

$$
\begin{gathered}
z= \pm \sqrt{\frac{\left(1+S_{11}\right)^{2}-S_{21}^{2}}{\left(1-S_{11}\right)^{2}-S_{21}^{2}}} \\
n=\frac{1}{k_{0} d_{\text {eff }}} \\
\left\{\operatorname{Im}\left[\ln \left(\frac{S_{21}}{1-S_{11} R_{01}}\right)\right]+2 m \pi-\mathrm{j} \operatorname{Re}\left[\ln \left(\frac{S_{21}}{1-S_{11} R_{01}}\right)\right]\right\}, \\
\varepsilon_{\mathrm{y}}=n / z, \\
\mu_{\mathrm{z}}=n \cdot z
\end{gathered}
$$

where $d_{\text {eff }}$ is the thickness of the virtual homogeneous slab, which replaces the real geometry thickness, $k_{0}$ is the free space wave-number of the incident wave and $R_{01}=(z-1) /(z+1)$. Here $m$ is an integer related to the branch index of real $(n)$ [18].

The transmission characteristics of the artificial material are verified by the Ansys HFSS. The magnitude and phase of the scattering parameters of the artificial material are depicted in Figs. 2(a) and (b), respectively. The reflection coefficient $\left(\mathrm{S}_{11}\right)$ is less than $-10 \mathrm{~dB}$ over $2-17 \mathrm{GHz}$, indicating that the energy can be transmitted through the artificial material within that frequency band. Here, the $\mathrm{S}$ shaped structure is providing longer current path compared to the parallel line non-resonant structure [14]. But the concept of non-resonant metamaterials can be obtained from simple, conducting elements. At a frequency, an electrical resonance occurs for the conductor length due 
to inductance and capacitance offered by the elements [19]. The resonant frequency of the proposed artificial material occurs at $16 \mathrm{GHz}$. The proposed material has a non-resonant characteristic over wide frequency band $2-13 \mathrm{GHz}$ as shown in Fig. 2(c). The normalized impedance of the artificial material is near to 1 within non-resonant region as

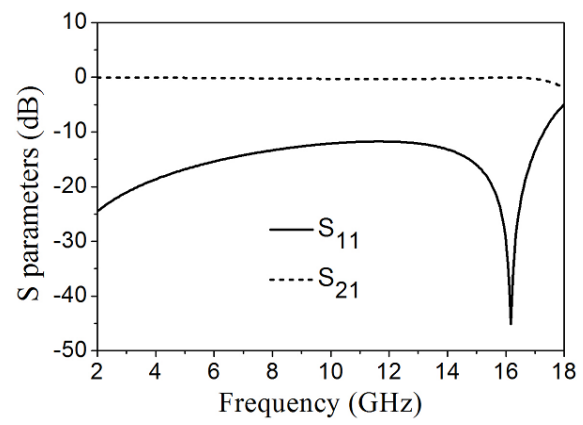

(a)

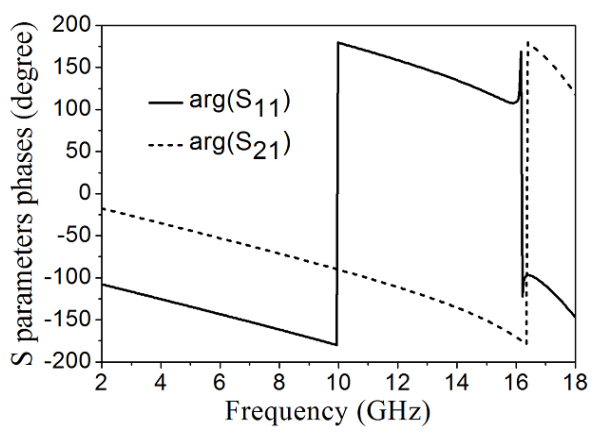

(b)

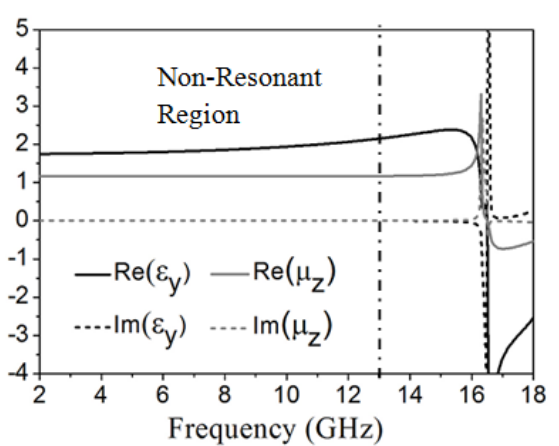

(c)

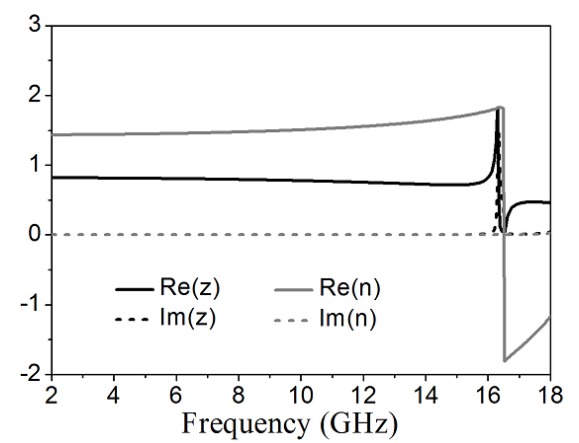

(d)

Fig. 2. The parameters of the unit cell artificial material: (a) magnitude of scattering parameters, (b) phases of scattering parameters, (c) the effective permittivity $\left(\varepsilon_{\mathrm{y}}\right)$ and permeability $\left(\mu_{\mathrm{z}}\right)$, (d) the normalized impedance $(z)$ and effective refractive index $(n)$. illustrated in Fig. 2(d). It means a good impedance match between the scattering object and air. The real and imaginary parts of refractive index are also presented in Fig. 2(d). The real part of the effective refractive index $(n)$ varies between 1.4-1.5 within non-resonant region and imaginary part is near to zero over non-resonant region.

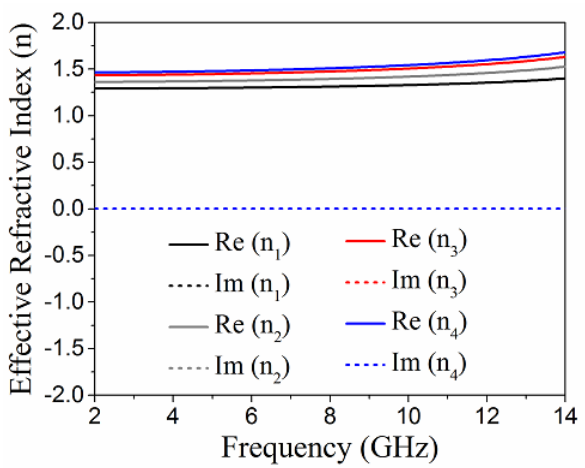

(a)

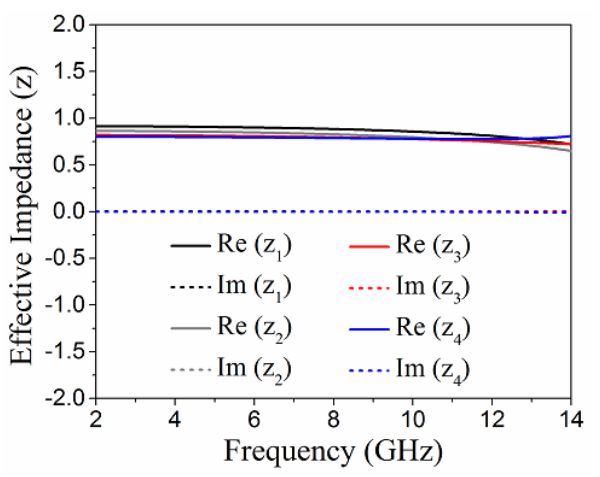

(b)

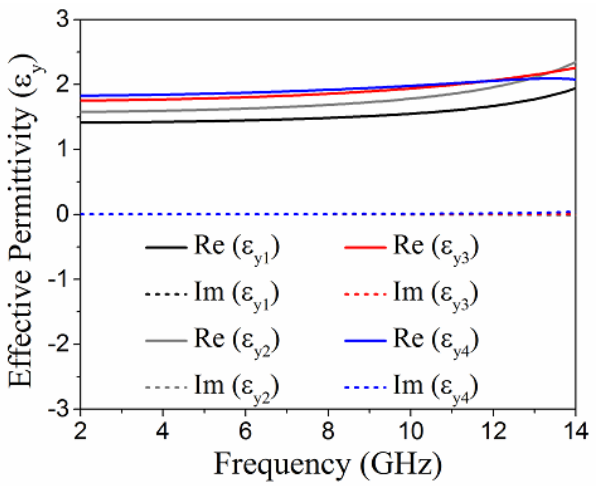

(c)

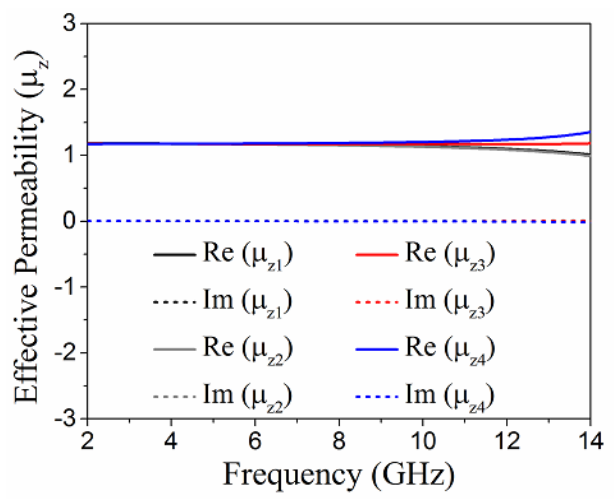

(d) 


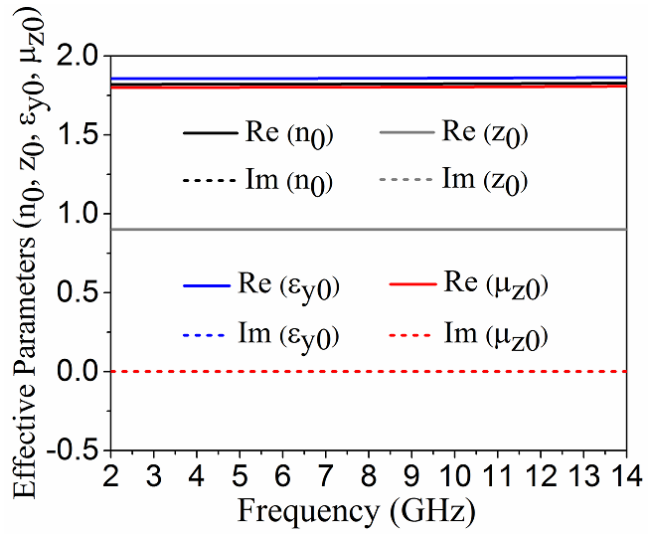

(e)

Fig. 3. The parameters for the different number of S-shaped structure in the unit cell artificial material: (a) effective refractive index $(n)$, (b) effective impedance $(z)$, (c) effective permittivity $\left(\varepsilon_{\mathrm{y}}\right),(\mathrm{d})$ effective permeability $\left(\mu_{z}\right)$, and (e) effective material parameters $\left(n_{0}, z_{0}, \varepsilon_{\mathrm{y} 0}\right.$ and $\left.\mu_{\mathrm{z} 0}\right)$ of the antenna without using metamaterials.

To understand the effects of the numbers of S structures in effective material parameters, the effective parameters for different numbers of S-shaped structures in unit cell are shown in Fig. 3(a) to (d). The effective refractive index, impedance, permittivity and permeability are denoted as $n_{i}, z_{i}, \varepsilon_{\mathrm{y} i}$ and $\mu_{\mathrm{z} i}$, respectively, where $i=1,2,3,4$ is a number of S-shapes in unit cell. So, it is clear that the effective refractive index and permittivity are enhanced by increasing the number of S-shape structures in unit cell. But the effective impedance is close to unity by decreasing the number of S-shape structures. Moreover, the effective permeability is almost constant over the frequency band 2 to $11 \mathrm{GHz}$. The simulations of the effective material parameters of the antenna without using metamaterials are shown in Fig. 3(e). The effective permittivity $\left(\varepsilon_{\mathrm{y} 0}\right)$ and permeability $\left(\mu_{\mathrm{z} 0}\right)$ are varying near at 1.79 to 1.82 , respectively over the frequency band 2 to $14 \mathrm{GHz}$. But the effective refractive index $\left(n_{0}\right)$ and impedance $\left(z_{0}\right)$ values are also very close to 1.8 and 1 , respectively. So, the EM wave propagates in antenna without using metamaterial whose refractive index is approximately 1.8 .

\section{Design of Tapered Slot Antenna}

The geometry of the basic tapered slot antenna structure is shown in Fig. 4. It is fabricated on Rogers RO4003 substrate with dielectric constant $\left(\varepsilon_{\text {ant }}\right) 3.55$, loss tangent $(\tan \delta) 0.0027$ and substrate thickness of $0.8 \mathrm{~mm}$. The dimensions of the basic TSA are about $40 \times 46 \times 0.8 \mathrm{~mm}^{3}$. The excitation of the antenna is given by using a microstrip line with impedance transition techniques to match $50 \Omega$ SMA connectors. The broader and smaller microstrip line widths are calculated as $W_{\mathrm{f} 2}=1.7 \mathrm{~mm}$ and $W_{\mathrm{f} 1}=0.8 \mathrm{~mm}$, respectively. The exponential inner curves adopted in this design can be described by the equation [16]

$$
y= \pm\left(c_{1} \mathrm{e}^{R x}+c_{2}\right)
$$

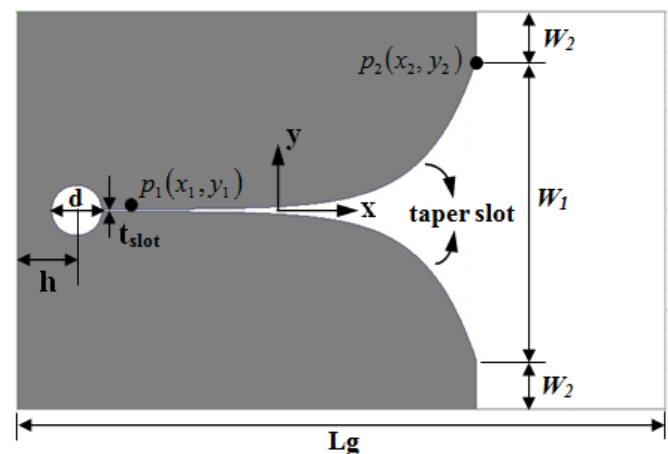

(a)

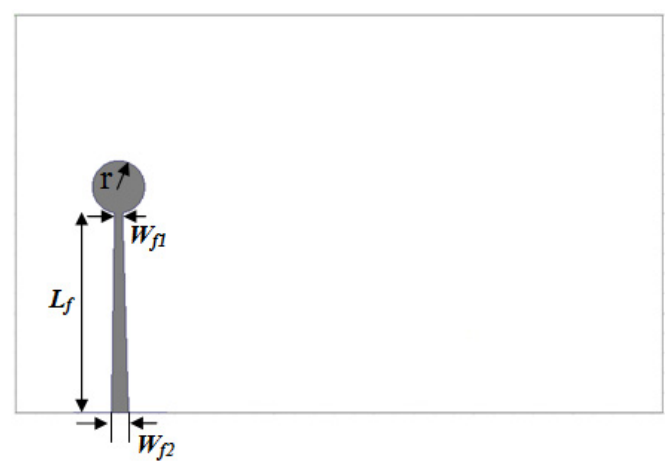

(b)

Fig. 4. The geometry of the basic TSA: (a) Top view, (b) bottom view.

\begin{tabular}{|c|c|c|c|}
\hline Parameters & Value $(\mathrm{mm})$ & Parameters & Value $(\mathrm{mm})$ \\
\hline$L \mathrm{~g}$ & 65 & $t_{\text {slot }}$ & 0.2 \\
\hline$W_{1}$ & 30 & $r$ & 2.6 \\
\hline$W_{2}$ & 5 & $L_{\mathrm{f}}$ & 20.4 \\
\hline$h$ & 6 & $L_{\mathrm{f} 1}$ & 0.8 \\
\hline$d$ & 5 & $L_{\mathrm{f} 2}$ & 1.7 \\
\hline
\end{tabular}

Tab. 1. The optimal parameters of the basic TSA.

where

$$
c_{1}=\frac{y_{2}-y_{1}}{\mathrm{e}^{R x_{2}}-\mathrm{e}^{R x_{1}}}, \quad c_{2}=\frac{y_{1} \mathrm{e}^{R x_{2}}-y_{2} \mathrm{e}^{R x_{1}}}{\mathrm{e}^{R x_{2}}-\mathrm{e}^{R x_{1}}} .
$$

The points $p_{1}\left(x_{1}, \pm y_{1}\right)$ and $p_{2}\left(x_{2}, \pm y_{2}\right)$ are shown in Fig. 4(a), where $R$ is the exponential rate which controls the shape of slot. From (5), the ranges of $x$ and $y$ axes of the slot are $2.5 \mathrm{~mm} \leq x \leq 37.5 \mathrm{~mm}$ and $0.1 \mathrm{~mm} \leq y$ $\leq 15 \mathrm{~mm}$, respectively.

The tapered slot antenna starts to radiate when its Eplane width $\left(W_{1}\right)$ is half-wavelength [20]. To improve impedance matching of the antenna, a matching section is included in feeding line. The microstrip to slotline transition is realized by etching the slotline on one side of a substrate. On the opposite side of the substrate, a microstrip line crosses perpendicular to the slotline. The microstrip line is closed by a circular radial stub which acts as a wideband short circuit. Moreover, the microstrip-slotline transition exhibits a frequency-independent transition characteristic of electromagnetic field [21]. The optimized dimensions of the basic TSA are shown in Tab. 1. 


\section{Design and Analysis of SRR Slot in Artificial Material TSA}

The SRR is used to create a band notch for the UWB TSA. According to the position of the SRR on the antenna, band notch characteristics will be affected. In case of basic TSA, the operation of the antenna depends on balun, microstrip feeding line. Area near to excitation or input aperture area of the taper slot is called transition-area. The geometry of SRR structure on the radiating patch is depicted in Fig. 5(a). The length of the substrate is extended up to $19 \mathrm{~mm}$ in the modified TSA to accommodate the metamaterial.

In order to understand the effects of SRR slot, it is placed at different positions namely P1, P2 and P3 locating

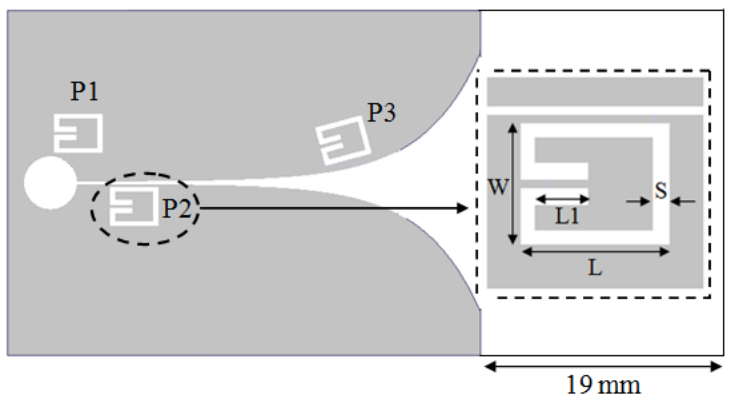

(a)

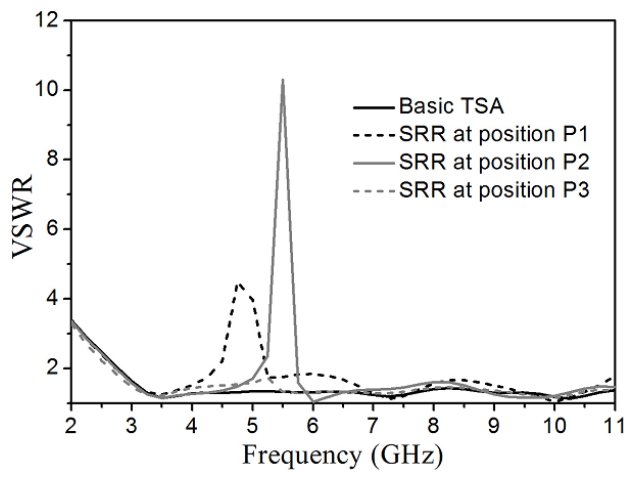

(b)

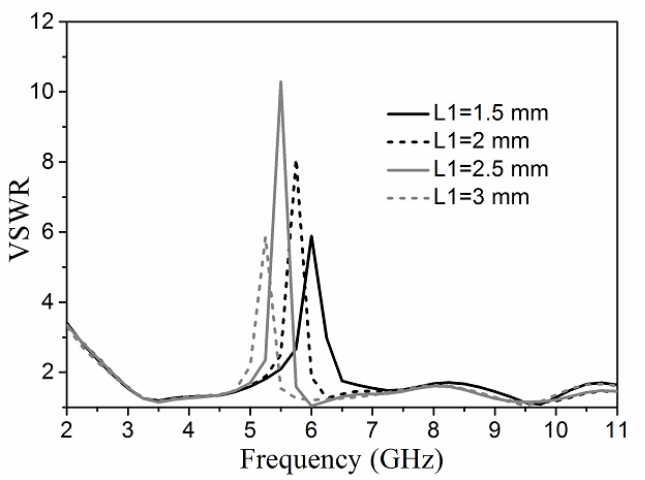

(c)

Fig. 5. The configuration of SRR slot in basic TSA: (a) radiating patch with SRR slot in different positions, (b) corresponding VSWR for SRRs slot in different positions, and (c) variation of VSWR for the different values of $\mathrm{L} 1$. The final dimensions of the SRR unit cell are $\mathrm{L}=5 \mathrm{~mm}, \mathrm{~L} 1=2.5 \mathrm{~mm}, \mathrm{~W}=3.5 \mathrm{~mm}, \mathrm{~S}=0.5 \mathrm{~mm}$. in the feeding-area, the transition-area, and the rest area of taper slot, respectively. The simulated VSWR of the basic TSA structure where SRR slots are placed at positions P1, P2 and P3 is presented in Fig. 5(b). It is clear that the basic TSA has a very good impedance matching from 3 to $11 \mathrm{GHz}$ and VSWR spikes at $4.8 \mathrm{GHz}, 5.5 \mathrm{GHz}$ for SRR positions $\mathrm{P} 1$ and $\mathrm{P} 2$, respectively. In case of $\mathrm{P} 3$ position, SRR is too far from excitation point. Therefore, the band notch does not work properly. Finally, the WLAN band notch is active, when SRR is etched at the position P2. The electric field is strongly coupled with SRR at the taper slot to create a strong resonance. So, the currents of all working bands are allowed except at the resonant frequency. As a result, a narrow band is suppressed within UWB frequency range. To understand the effects of current path, the parameter L1 of the SRR slot has been varied as $1.5 \mathrm{~mm}, 2 \mathrm{~mm}, 2.5 \mathrm{~mm}$ and $3 \mathrm{~mm}$. The VSWR plot is also shown in Fig. 5(c). It can be seen that the notches are created at $6 \mathrm{GHz}, 5.8 \mathrm{GHz}, 5.5 \mathrm{GHz}$ and $5.2 \mathrm{GHz}$ for the different values of L1.

In order to understand the effect of artificial material, the proposed material is placed in front of the taper slot area in a periodic fashion. The artificial material loaded TSA with SRR is depicted in Fig. 6(a). The VSWR comparison of the basic TSA, SRR etched TSA with and without artificial material is shown in Fig. 6(b). From this,

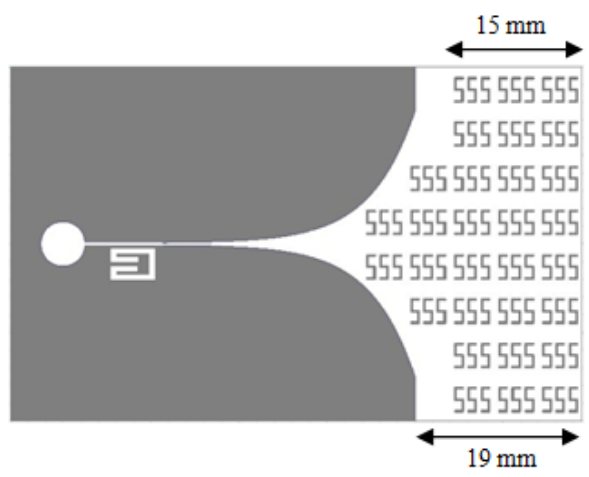

(a)

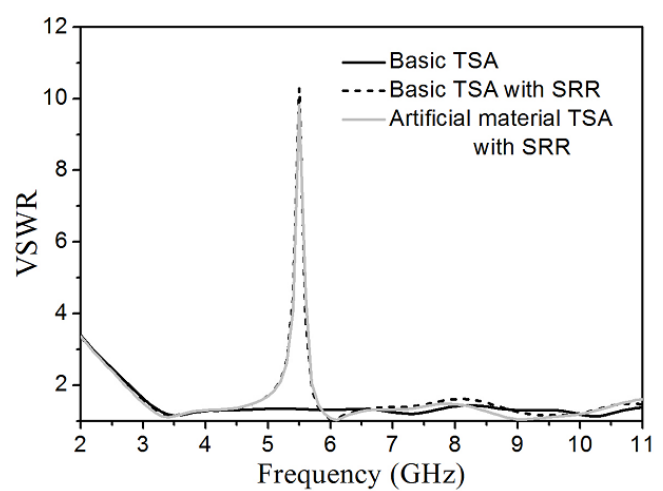

(b)

Fig. 6. The configuration of artificial material integrated TSA with SRR slot: (a) Artificial material TSA, (b) comparison of VSWR simulated result for basic TSA, basic TSA with SRR and artificial material TSA with SRR. 
it is observed that the VSWR is not affected by using artificial material in basic TSA. So, it is considered that the VSWR for both antennas are notched at the WLAN band.

In order to understand the effect of the lens, the electric field distribution of the TSA without and with artificial material is compared as shown in Figs. 7(a) and (b), respectively. So, it is clear that the electric field intensity of the TSA without artificial material is comparatively lower than that of artificial material loaded TSA. So, EM wave can travel more distance by using artificial material. The surface current distributions of the artificial material loaded TSA with SRR at position P2 is also shown in Fig. 7(c). It is clear that the current density is more in the SRR region at $5.5 \mathrm{GHz}$, it means the EM wave is suppressed particularly at that frequency.

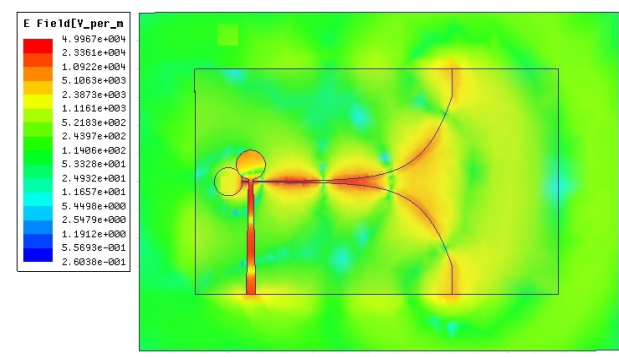

(a)

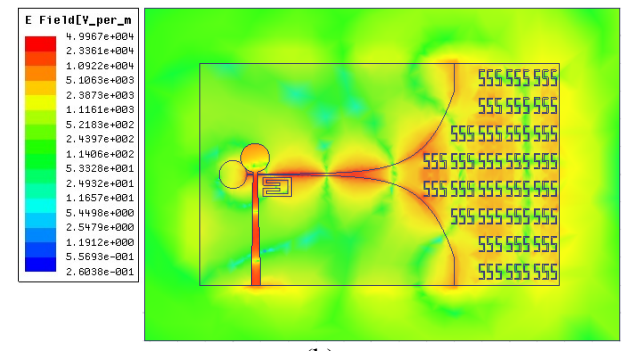

(b)

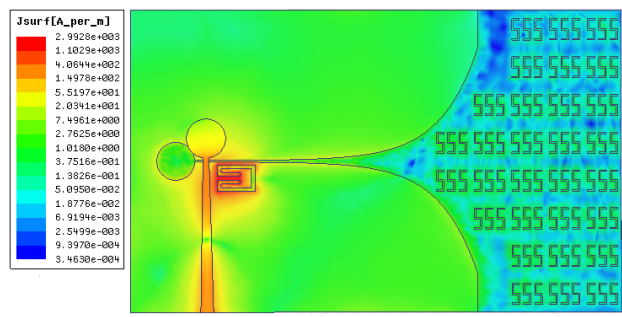

(c)

Fig. 7. The field distribution at $10 \mathrm{GHz}$ : (a) Basic TSA, (b) artificial material cells integrated TSA, and (c) the magnitude of current distribution at $5.5 \mathrm{GHz}$ of the artificial material loaded TSA with SRRs at P2 position.

According to the physics, the phase velocity $\left(v_{\mathrm{ph}}\right)$ of any medium is inversely proportional to the refractive index of that medium $\left(v_{\mathrm{ph}}=c / n\right.$ where $n$ is the refractive index of the medium). In case of basic TSA, the EM wave is propagating on tapered slot with constant phase velocity and refractive index of antenna substrate $n_{\text {ant }}=\sqrt{\varepsilon_{\text {ant }}}=1.88$.

From Fig. 3(e), the simulated effective refractive index of the antenna is also varying near to 1.8 without using meta- materials. But, the refractive index in the artificial material area is approximately 1.5 over frequency band 2 to $12 \mathrm{GHz}$ which is lower than the refractive index of antenna ( $\left.n_{\text {ant }}\right)$. Hence, it is clear that the phase velocity of the EM wave in the proposed metamaterial region is much greater than the antenna substrate. Hence, the radiated beam of the basic TSA will be more directive with narrow beam width, when EM wave enters the artificial material region. In this way the proposed material behaves like a beam focusing lens.

\section{Results and Discussion}

The top and bottom views of the fabricated basic TSA and artificial material loaded TSA with SRR slot are shown in Fig. 8. The comparison between the proposed antenna and published works in terms of size, operating frequency band, peak gain at notch frequency and rejection band application are listed in Tab. 2. The proposed antenna has better size reduction compared to the existing structures which is mentioned in Tab. 2. It can be noticed that the gain level is positive at the notched frequency band in [16]. Therefore, the radiation of the antenna is not suppressed at the notch frequency.

The measured VSWR of basic TSA and artificial material TSA fluctuating at 1.5 over frequency band 3 to $11 \mathrm{GHz}$, is illustrated in Fig. 9, indicating the good impedance matching except at 5.1-5.8 GHz. It can be seen that the VSWR of the basic TSA and artificial material TSA are spiking up to 10 and 7 at $5.5 \mathrm{GHz}$, respectively. VSWR of the both antennas overlap at the notch frequency band. So, it is confirmed that the WLAN band is suppressed by using SRR.

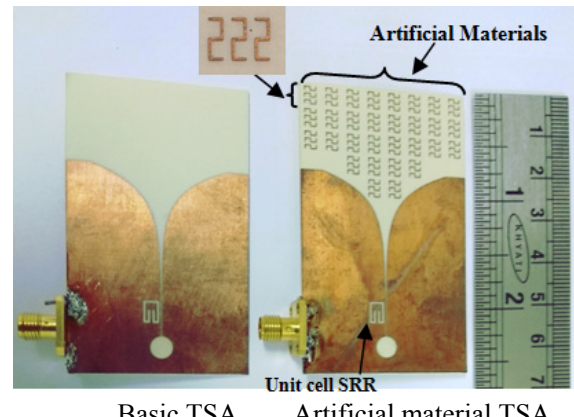

(a)

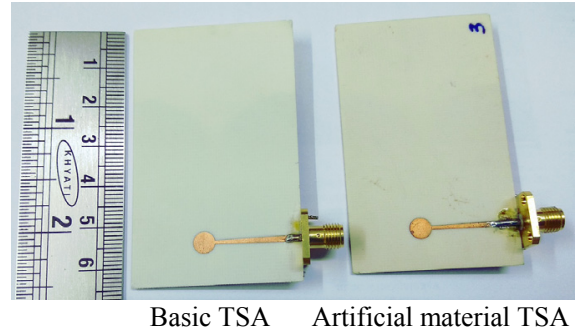

(b)

Fig. 8. The photographs of the fabricated basic TSA and artificial material TSA with SRR: (a) Top view, (b) bottom view. 


\begin{tabular}{|c|c|c|c|c|c|}
\hline Ref. & Size $\left(\mathbf{m m}^{\mathbf{2}}\right)$ & $\begin{array}{c}\text { Operating } \\
\text { frequency band }\end{array}$ & $\begin{array}{c}\text { Gain level at } \\
\text { notch frequency }\end{array}$ & Peak gain & $\begin{array}{c}\text { Rejected band } \\
\text { application }\end{array}$ \\
\hline$[10]$ & $80 \times 80$ & $3-12 \mathrm{GHz}$ & $-2 \mathrm{~dB}$ & $8 \mathrm{~dB}$ & $\mathrm{n} / \mathrm{a}$ \\
\hline$[16]$ & $50 \times 50$ & $3.1-10.6 \mathrm{GHz}$ & $2.6 \mathrm{dBi}$ & $8.5 \mathrm{dBi}$ & $\mathrm{WLAN}$ \\
\hline$[13]$ & $80 \times 140$ & $3-14 \mathrm{GHz}$ & $\mathrm{n} / \mathrm{a}$ & $14 \mathrm{~dB}$ & $\mathrm{n} / \mathrm{a}$ \\
\hline$[14]$ & $42 \times 72$ & $6-19 \mathrm{GHz}$ & $\mathrm{n} / \mathrm{a}$ & $12 \mathrm{dBi}$ & $\mathrm{n} / \mathrm{a}$ \\
\hline Proposed antenna & $40 \times 65$ & $3-11 \mathrm{GHz}$ & $-1.2 \mathrm{dBi}$ & $11 \mathrm{dBi}$ & $\mathrm{WLAN}$ \\
\hline
\end{tabular}

Tab. 2. Comparison between proposed antenna and existed work.

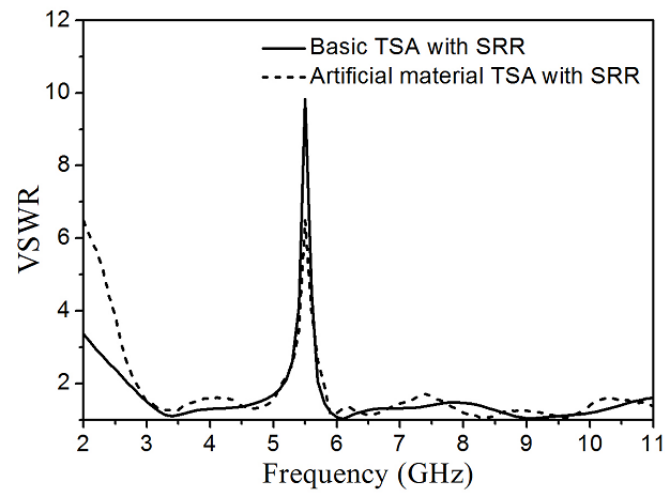

Fig. 9. The measured VSWR of the basic TSA and artificial material TSA.

The normalized radiation patterns (E-plane) of the fabricated TSA at $6.5 \mathrm{GHz}, 8.5 \mathrm{GHz}$, and $10 \mathrm{GHz}$ are shown in Fig. 10. The beam width of the artificial material integrated TSA is much less than basic TSA, which indicates that the pattern becomes more directive. The measurement results of the peak gain with respect to the frequency graph of the both antennas are shown in Fig. 11(a). Compared to the low frequency region, the measured gain is effectively increased at high frequency side. Hence, it is clear that the maximum gain of the basic TSA is increased

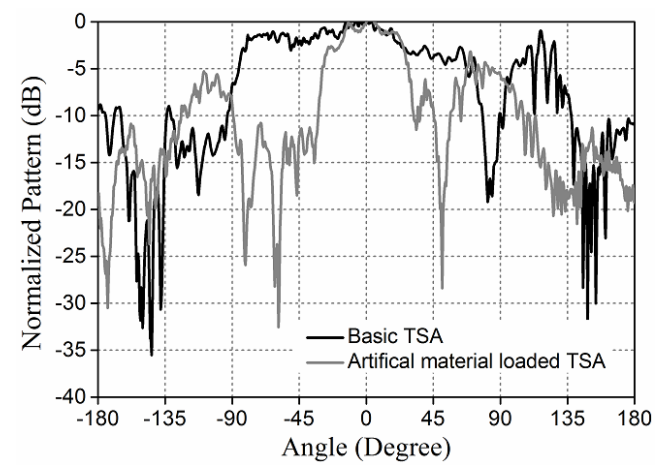

(a)

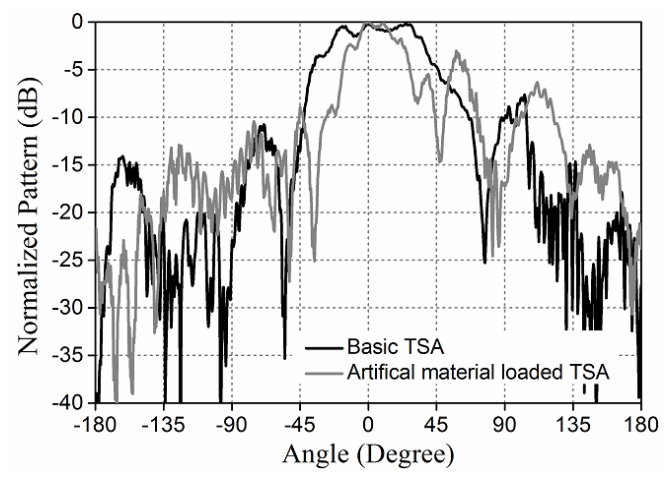

(b)

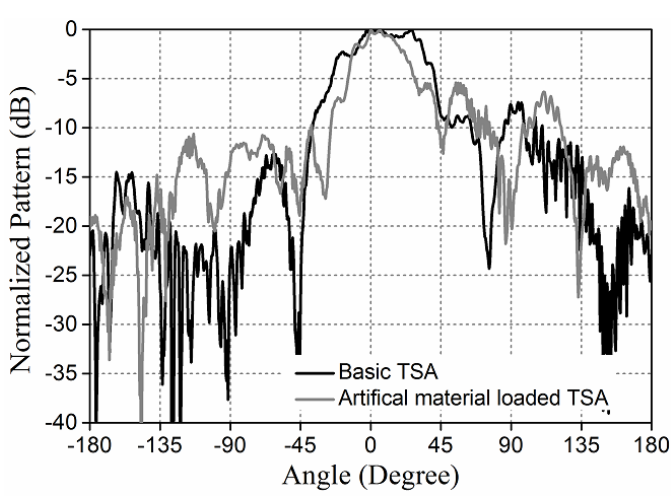

(c)

Fig. 10. Measured E-plane normalized radiation pattern of basic TSA and artificial material TSA at (a) $6.5 \mathrm{GHz}$, (b) $8.5 \mathrm{GHz}$, (c) $10 \mathrm{GHz}$.

from 9.4 to $10.9 \mathrm{dBi}$ at $10 \mathrm{GHz}$ (approximately). Obviously, it can be noticed that the peak gain of the basic TSA and artificial material loaded TSA with SRR slot is decreased up to $-4 \mathrm{dBi}$ at $5.5 \mathrm{GHz}$.

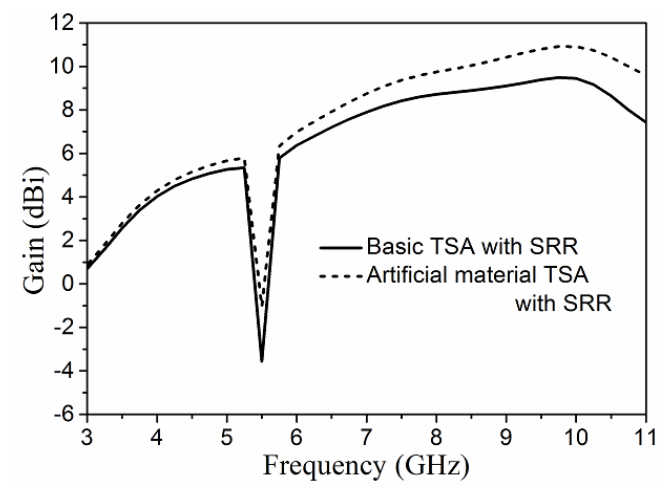

(a)

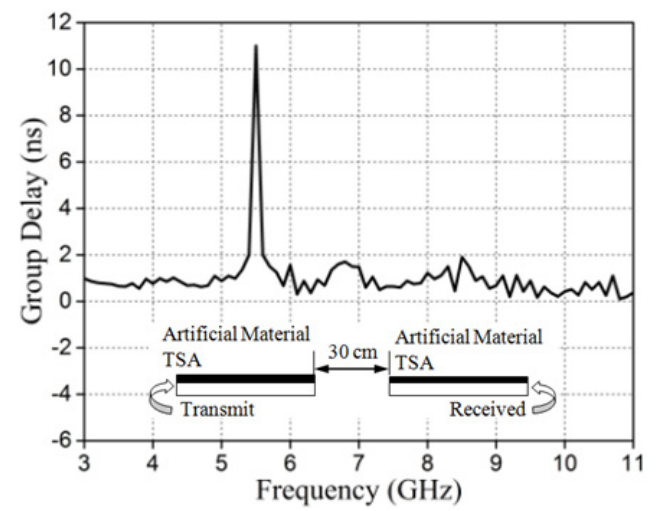

(b)

Fig. 11. Measured results: (a) Peak gain of basic TSA and artificial material TSA. (b) Group delay for the artificial material TSA with SRR. 
To understand the time domain performance of the UWB antenna, the time domain characteristic of the fabricated artificial material integrated TSA is tested. In order to measure group delay, two antennas are positioned side by side with a distance $30 \mathrm{~cm}$ and connected through a vector network analyzer (VNA). The antenna group delay is fluctuating within the range of approximately $\pm 1.2 \mathrm{~ns}$ in the desired band as shown in Fig. 11(b). Hence, it can be considered as stable. It indicates that a transmitted signal will not be seriously distorted by the proposed antenna except at notched frequency.

\section{Conclusion}

This paper introduces a broadband artificial material which is integrated with the UWB TSA to improve the radiation pattern and gain of the antenna. SRR cell is used to suppress the WLAN band in the TSA. The band rejection at $5.5 \mathrm{GHz}$ of the artificial material TSA has been successfully done by using unit cell SRR. The measured results of the fabricated antenna are showing that the gain is increased by $1.5 \mathrm{dBi}$ with narrow beam width. Therefore, the proposed antenna is a good candidate for ultrawideband communication systems with a notch at specific frequency band.

\section{References}

[1] GIBSON, P. J. The Vivaldi aerial. In 9th European Microwave Conference. Brighton (UK), 1979, p. 101-105. DOI: 10.1109/EUMA.1979.332681

[2] REID, E. W., ORTIZ-BALBUENA, L., GHADIRI, A., MOEZ, K. A 324 element Vivaldi antenna array for radio astronomy instrumentation. IEEE Transactions on Instrumentation and Measurement, 2012, vol. 61, no. 1, p. 241-249. DOI: 10.1109/TIM.2011.2159414

[3] ELLIS, T. J., REBEIZ, G. M. MM-wave tapered slot antennas on micromachined photonic bandgap dielectrics. In IEEE MTT-S International Microwave Symposium Digest. San Francisco (CA, USA), 1996, p. 1157-1160. DOI: 10.1109/MWSYM.1996.511235

[4] JAYASINGHE, J. M. J. W., ANGUERA, J., UDUWAWALA, D. $\mathrm{N}$. Genetic algorithm optimization of a high-directivity microstrip patch antenna having a rectangular profile. Radioengineering, 2013 , vol. 22 , no. 3 , p. $700-707$.

[5] SONG, K., LEE, S.-H., KIM, K., HUR, S., KIM, J. Emission enhancement of sound emitters using an acoustic metamaterial cavity. Scientific Report, 2014, vol. 4, no. 4165, 6 p. DOI: 10.1038/srep04165

[6] JIANG, W. X., QIN, C. W., HAN, T. C., et al. Broadband alldielectric magnifying lens for far-field high-resolution imaging. Advanced Materials, 2013, vol. 25, p. 6963-6968. DOI: 10.1002/adma.201303657

[7] LANDY, N., SMITH, D. R. A full-parameter unidirectional metamaterial cloak for microwaves. Nature Materials, 2013, vol. 12 , p. $25-28$. DOI: $10.1038 /$ nmat3476

[8] R. LIU, R., CHENG, Q., HAND, T., et al. Experimental demonstration of electromagnetic tunneling through an epsilonnear-zero metamaterial at microwave frequencies. Physical Review
Letters, 2008, vol. 100, no. 2, p. 023903-1-023903-4. DOI: 10.1103/PhysRevLett.100.023903

[9] CAO, T., WEI, C. W., SIMPSON, R. E., et al. Broadband polarization-independent perfect absorber using a phase-change metamaterial at visible frequencies. Scientific Report, 2014, vol. 4, no. 3955,8 p. DOI: $10.1038 /$ srep03955

[10] LIU, X., LEI, Z., YANG, R., et al. The band notch sensitivity of Vivaldi antenna towards CSRRs. Progress In Electromagnetics Research Letters, 2013, vol. 43, p. 125-135. DOI: 10.2528/PIERL13080611

[11] SIDIQUI, J. Y., SAHA, C., ANTAR, Y. M. M. Compact SRR loaded UWB circular monopole antenna with frequency notch characteristics. IEEE Transactions on Antennas and Propagation, 2014, vol. 62, no. 8, p. 4015-4020. DOI: 10.1109/TAP.2014.2327124

[12] RANGA, Y., MATEKOVITS, L., ESSELle, K. P. et al. Multioctave frequency selective surface reflector for ultrawideband antennas. IEEE Antennas and Wireless Propagation Letters, 2011, vol. 10, p. 219-222. DOI: 10.1109/LAWP.2011.2130509

[13] ZHOU, B., CUI, T. J. Directivity enhancement to Vivaldi antennas using compactly anisotropic zero-index metamaterials. IEEE Antennas and Wireless Propagation Letters, 2011, vol. 10, p. 326 to 329. DOI: 10.1109/LAWP.2011.2142170

[14] CHEN, L., LEI, Z., YANG, R., et al. A broadband artificial material for gain enhancement of antipodal tapered slot antenna. IEEE Transactions on Antennas and Propagation, 2015, vol. 63, no. 1, p. 395-400. DOI: 10.1109/TAP.2014.2365044

[15] ZHU, F., GAO, S., HO, A. T. S., et al. Miniaturized tapered slot antenna with signal rejection in $5-6-\mathrm{GHz}$ band using a balun. IEEE Antennas and Wireless Propagation Letters, 2012, vol. 11, p. 507-510. DOI: 10.1109/LAWP.2012.2199276

[16] BHASKAR, M., JOHARI, E., AKHTER, Z., et al. Gain enhancement of the Vivaldi antenna with band notch characteristics using zero-index metamaterial. Microwave and Optical Technology Letters, 2016, vol. 58, no. 1, p. 233-238. DOI: 10.1002/mop. 29534

[17] SAHA, C., NATANI, P., SHAIK, L. A., et al. Square/hexagonal split ring resonator loaded exponentially tapered slot ultra wideband (UWB) antenna with frequency notch characteristics. Microwave and Optical Technology Letters, 2017, vol. 59, no. 6, p. 1241-1245. DOI: 10.1002/mop.30511

[18] CHEN, X., GREZEGORCZYK, T. M., WU, B.-I., et al. Robust method to retrieve the constitutive effective parameters of metamaterials. Physical Review E, 2004, vol. 70, p. 016608-1 to 016608-7. DOI: 10.1103/PhysRevE.70.016608

[19] LIU, R., CHENG, Q., CHIN, J. Y., et al. Broadband gradient index microwave quasi-optical elements based on non-resonant metamaterials. Optics Express, 2009, vol. 17, no. 23, p. 21030-21041. DOI: $10.1364 / \mathrm{OE} .17 .021030$

[20] GAZIT, E. Improved design of the Vivaldi antenna. Institution of Electrical Engineers Proceedings, 1988, vol. 135, no. 2, p. 89-92. DOI: $10.1049 /$ ip-h-2.1988.0020

[21] SCHUPPERT, B. Microstrip/slotline transition: modeling and experimental investigation. IEEE Transactions on Microwave Theory and Techniques, 1988, vol. 36, no. 8, p. $1272-1282$. DOI: $10.1109 / 22.3669$

\section{About the Authors ...}

Rahul SINGHA is born in Agartala, India in 1988. He received his B.E. in Electrical and Electronics Engineering and M.Tech. in Microelectronics \& VLSI Design from the 
National Institute of Technology (NIT) Agartala in 2010 and 2013, respectively. He is currently a Ph.D. student at the Dept. of Electronics and Communication Engineering, NIT Warangal. His research interests include metamaterial, UWB antenna design, microstrip filter.

Vakula DAMERA received her Bachelor's degree in Electronics and Communication Engineering from Nagarjuna University, AP, India, and Master's degree .Tech. from Birla Institute of Technology, Mesra, India, with
Microwave specialization in 1992 and 1994, respectively. She obtained Ph.D. on Fault Diagnostics of Antenna Arrays from the National Institute of Technology, Warangal, in 2010. She has been working as assistant professor at the National Institute of Technology, Warangal, India since 2006. She has published 31 papers in international conferences and journals. Her areas of interests include phase array antennas, ultra-wideband antennas, multiband antennas, fault diagnostics, and neural networks. 